\title{
Multiple exposures of sevoflurane during pregnancy induces memory impairment in young female offspring mice
}

\author{
Woosuk Chung ${ }^{1,2}$, Seunghwan Yoon ${ }^{1}$, and Yong Sup Shin ${ }^{1,2}$ \\ Department of Anesthesiology and Pain Medicine, ${ }^{1}$ Chungnam National University Hospital, ${ }^{2}$ Chungnam National \\ University College of Medicine, Daejeon, Korea
}

Background: Earlier studies have reported conflicting results regarding long-term behavioral consequences after anesthesia during the fetal period. Previous studies also suggest several factors that may explain such conflicting data. Thus, we examined the influence of age and sex on long-term behavioral consequences after multiple sevoflurane exposures during the fetal period.

Methods: C57BL/6J pregnant mice received oxygen with or without sevoflurane for 2 hours at gestational day (GD) 14-16. Offspring mice were subjected to behavioral assays for general activity (open field test), learning, and memory (fear chamber test) at postnatal day 30-35.

Results: Multiple sevoflurane exposures at GD 14-16 caused significant changes during the fear chamber test in young female offspring mice. Such changes did not occur in young male offspring mice. However, general activity was not affected in both male and female mice.

Conclusions: Multiple sevoflurane exposures in the second trimester of pregnancy affects learning and memory only in young female mice. Further studies focusing on diverse cognitive functions in an age-, sex-dependent manner may provide valuable insights regarding anesthesia-induced neurotoxicity.

Key Words: Fetus, Learning, Memory, Pregnancy, Sevoflurane, Toxicity.

\section{Introduction}

After the first report of unintentional neural damage follow-

Corresponding author: Yong Sup Shin, M.D., Ph.D.

Department of Anesthesiology and Pain Medicine, Chungnam National University College of Medicine, 282, Munhwa-ro, Jung-gu, Daejeon 35015, Korea

Tel: 82-42-280-7840, Fax: 82-42-280-7968

Email: ysshin@cnu.ac.kr

ORCID: https://orcid.org/0000-0001-5071-734X

Received: July 7, 2017.

Revised: August 7, 2017 (1st); August 17, 2017 (2nd).

Accepted: August 21, 2017.

Korean J Anesthesiol 2017 December 70(6): 642-647 https://doi.org/10.4097/kjae.2017.70.6.642 ing anesthesia during childhood, there is now a large amount of data regarding neurotoxicity in the developing brain $[1,2]$. While it is relieving to know that recent clinical studies suggest that a single, short exposure may not affect neurodevelopment [3,4], there is still not enough evidence to rule out the possibility of the negative effects of anesthesia on neuro development. Accordingly, the FDA has recently reported two safety announcements focusing on multiple, lengthy sedation or general anesthesia in children and pregnant women (https://www.fda.gov/ Drugs/DrugSafety/ucm532356.htm). However, Andropoulos and Greene [5] have also reported concerns of such announcements, since it may also delay necessary surgical and diagnostic procedures that require anesthesia, resulting in adverse outcomes for patients [5].

There are very few clinical studies focusing on the effects

(c) This is an open-access article distributed under the terms of the Creative Commons Attribution Non-Commercial License (http://creativecommons.org/ licenses/by-nc/4.0/), which permits unrestricted non-commercial use, distribution, and reproduction in any medium, provided the original work is properly cited. 
of anesthesia in the fetal brain. Immense neurodevelopment, such as neurogenesis and neural migration, begins eight weeks after conception and continues throughout the second trimester of pregnancy [6]. Since these processes are regulated through GABA and glutamate, it is possible that anesthetic agents may disrupt this highly regulated process [7]. Studies in gestational day (GD) 14-20 pregnant mice, which represent the late-first or early-second trimester of human pregnancy (http://www.translatingtime.net/home) [8], report conflicting results regarding long-term behavioral consequences after fetal anesthetic exposure [9-12]. Unfortunately, there is little understanding on why such conflicting results exist.

Among the many factors that may contribute to the conflicting results regarding long-term behavioral consequences from fetal anesthesia, we first focused on the age of mice at which behavioral assays were actually performed, based on the fact that behavioral consequences after fetal anesthesia were mostly reported in young postnatal day (PND) 30 mice, while older mice do not show differences [7,11-14]. We also examined how sexdependent neurodevelopment influences neurotoxicity during fetal anesthesia by separately comparing young male and female offspring mice $[15,16]$. Thus, in order to evaluate the importance of age and sex when studying the long-term consequences of early anesthesia during pregnancy, pregnant mice were exposed to sevoflurane as previously described and fear chamber tests were conducted with male and female offspring mice at PND 30-35 [12].

\section{Materials and Methods}

\section{Animals}

All experiments were performed after being approved by the Committees on Animal Research in our institute (no. 014A0009). C57BL/6J mice were housed in a regulated environment $\left(22^{\circ} \mathrm{C}, 24\right.$ hour light cycle) and had access to food and water ad libitum. Mice were weaned at 3 weeks and caged in groups of three to five.

\section{Anesthesia}

Sevoflurane exposure was performed as previously described [12]. Briefly, pregnant female mice (GD 14) were randomly divided into 2 groups. Mice in the control group were placed in a 1-1 plastic chamber and exposed to $100 \%$ oxygen $(4 \mathrm{~L} / \mathrm{min})$ for 2.5 hours for 3 consecutive days (GD 14-16). Sevoflurane group mice were treated identically, except that $2.5 \%$ sevoflurane was added during the first two hours. The anesthesia chamber was placed in a water bath $\left(36^{\circ} \mathrm{C}\right)$ for temperature control and gas was analyzed using a $\mathrm{S} / 5$ compact anesthetic monitor and an e-
CAiO gas analyzer module (Datex-Ohmeda, Helsinki, Finland).

\section{Behavior tests}

Behavioral tests were performed with two cohorts of offspring mice obtained from eight pregnant mice that received multiple exposures of oxygen or sevoflurane (four mice each). The open field test and fear chamber test were performed as previously described with both male and female offspring at PND $30-35[12,17,18]$. There was at least a one day resting period between tests. All tests were recorded and later analyzed with a tracking software (Ethovision XT, Noldus Information Technology, Waxgeningen, the Netherlands \& Freezeframe, Actimetrics, Wilmette, IL, USA).

\section{Open field test}

General activity was evaluated after placing the mice in the center of a white cylinder (diameter $30 \mathrm{~cm}$, height $40 \mathrm{~cm}$ ). Locomotion was analyzed for $60 \mathrm{~min}$ with a tracking software (Ethovision).

\section{Fear test}

The fear test was conducted with a fear chamber (Coulbourn Instruments, Holliston, MA, USA) as previously described. Fear conditioning was performed 5-minutes after placing mice in the fear chamber: three trials of a conditioned stimulus (CS, 20 -second long, $3 \mathrm{kHz}, 80 \mathrm{~dB}$ tone) paired with an unconditioned stimulus (US, 1 second long, $0.5 \mathrm{~mA}$ electrical shock) at a 60 -second interval. Contextual-fear memory was measured 24 hours later after being placed in the same chamber for $5 \mathrm{~min}$ utes. Cue-memory was analyzed after another 24 hours by placing the mice in a novel chamber. CS was applied for 3 minutes after a 5-minute habituation period.

\section{Statistical analysis}

Statistical software R (3.1.2; R Core Team, Vienna, Austria) was used for analysis as previously described $[12,17,18]$. Continuous variables were tested for the assumption of normality and homogeneity of variance. Kruskal-Wallis test, Whelch's $t$ test, and t test were performed accordingly. Welch's $t$ test was performed when homogeneity of variance was unmet, and the Kruskal-Wallis test was performed if normality was unmet. Student's $t$ test was performed only when both conditions were met. Mixed effect modeling was used for analyzing repeatedly measured data (fear conditioning during the fear chamber test). Differences with $\mathrm{P}<0.05$ were considered statistically significant. 


\section{Results}

In order to evaluate the possibility that sevoflurane exposure during pregnancy induces age-dependent learning and memory impairments, we performed the fear chamber test in PND 30-35 mice. Previous studies regarding neurotoxicity during the fetal period have reported long-term behavioral impairments in this particular age, while several other studies focusing on older mice (over eight weeks) showed normal cognitive function. However, young mice that received multiple sevoflurane exposures (sevoflurane group) displayed similar learning and memory throughout the fear chamber test (Fig. 1). Fetal exposures to sevoflurane did not affect fear conditioning in young offspring mice (Fig. $1 \mathrm{~A})$, which is used to examine learning ability by analyzing the increase of fear behavior during three trials of a CS (20-s long, $3 \mathrm{kHz}, 80 \mathrm{~dB}$ tone) and an US (1 mV shock) [12]. Long-term memory was assessed by measuring contextual-, cue-memory during the 2 following days [12]. Contextual-fear memory (measuring freezing behavior while exposing the mice to the same fear chamber) and cue-fear memory (measuring freezing behavior in a new context after applying a CS) were also not affected from fetal sevoflurane exposures in young offspring mice (Figs. $1 \mathrm{~B}$ and $1 \mathrm{C}$ ).

Sex has also been implied to be an important factor for anesthetic neurotoxicity $[15,16]$. Thus, in order to exclude sexual differences, we performed additional analysis after separating male and female mice. Both male and female mice show normal learning during fear conditioning (Figs. 2A and 2D). Interestingly, while male mice show normal long-term memory (Figs. $2 \mathrm{~B}$ and $2 \mathrm{C}$ ), sevoflurane exposed female mice display significant changes in fear memory compared to control group female mice (Figs. 2E and 2F). Fetal sevoflurane exposures significantly impaired contextual-fear memory in young females (Fig. 2E). Unexpectedly, cue-fear memory was significantly increased in young female mice who received early anesthesia (Fig. 2F). The difference between male and female mice was not due to differences in general activity, as shown in the open field test (Fig. 3).

\section{Discussion}

With the growing awareness of potential negative effects after anesthesia in children and pregnancy, many parents and health givers express concerns of possible long-term neurodevelopmental complications after anesthesia during childhood. Even though recent studies suggest that a short, single episode of general anesthesia does not affect neurodevelopment $[3,4]$, there still remains the possibility of negative effects after multiple or lengthy exposure to anesthetics [19]. We have recently reported that both single and multiple sevoflurane exposures during the fetal period does not affect behavior at an adult age in male mice [12]. Our results may cause confusion, since they do not agree with previous studies that used the same anesthetic procedure $[9,10]$. On the other hand, by understanding the cause of such conflicting results can help us gain valuable insight regarding anesthesia-induced neurotoxicity.

We first focused on the actual age at which behavior was assessed. We considered age as an important factor based on several facts. Most studies that show negative long-term behavioral consequences from fetal anesthesia were performed at PND 30, while studies performed at a later age reported no differences
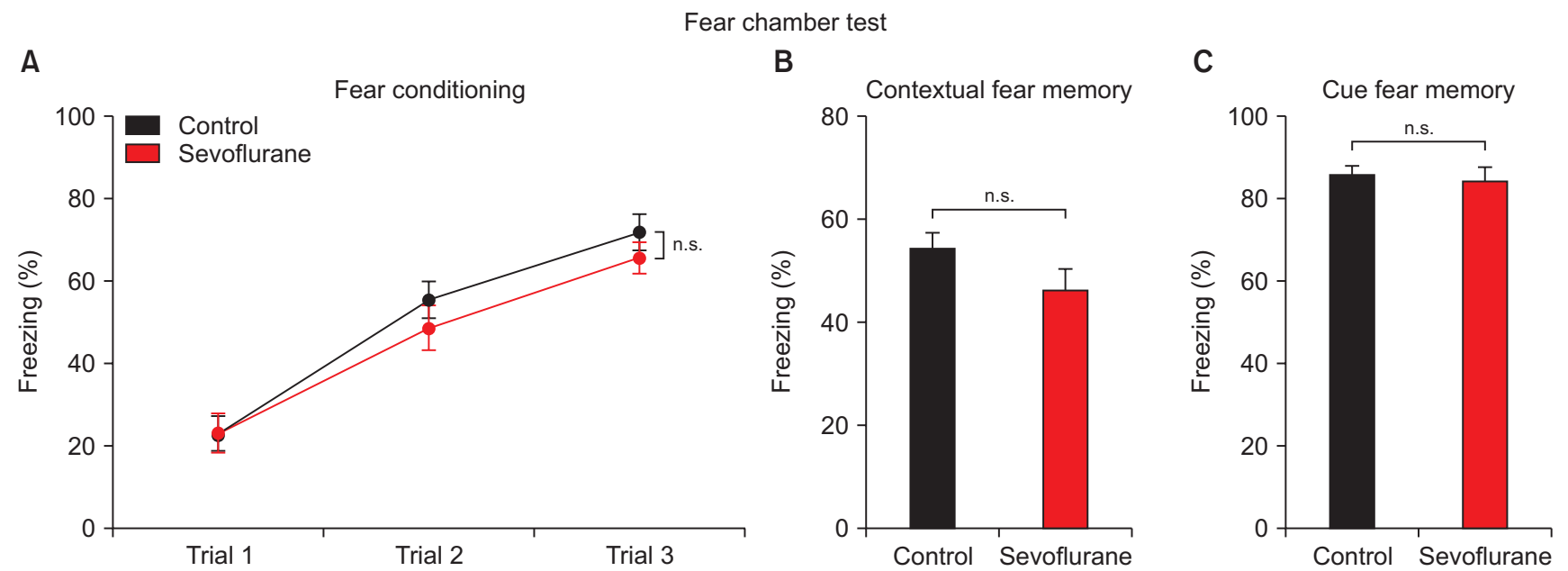

Fig. 1. Multiple sevoflurane exposures during pregnancy do not affect learning and memory in young offspring mice. (A) Sevoflurane exposed mice show a similar increase of freezing behavior during fear conditioning (linear regression was used to analyze the interaction between groups according to trials, $\mathrm{P}=0.491$; ns, not significant). (B, C) Both contextual and cue fear memorise are not affected by sevoflurane exposures during pregnancy (student's $\mathrm{t}$ test for contextual fear memory, $\mathrm{P}=0.098$; Kruskall-Wallis test for cue fear memory, $\mathrm{P}=0.976$; ns, not significant). $\mathrm{N}=21,24$ for control and sevoflurane groups, respectively. Values are presented as mean \pm SEM. 

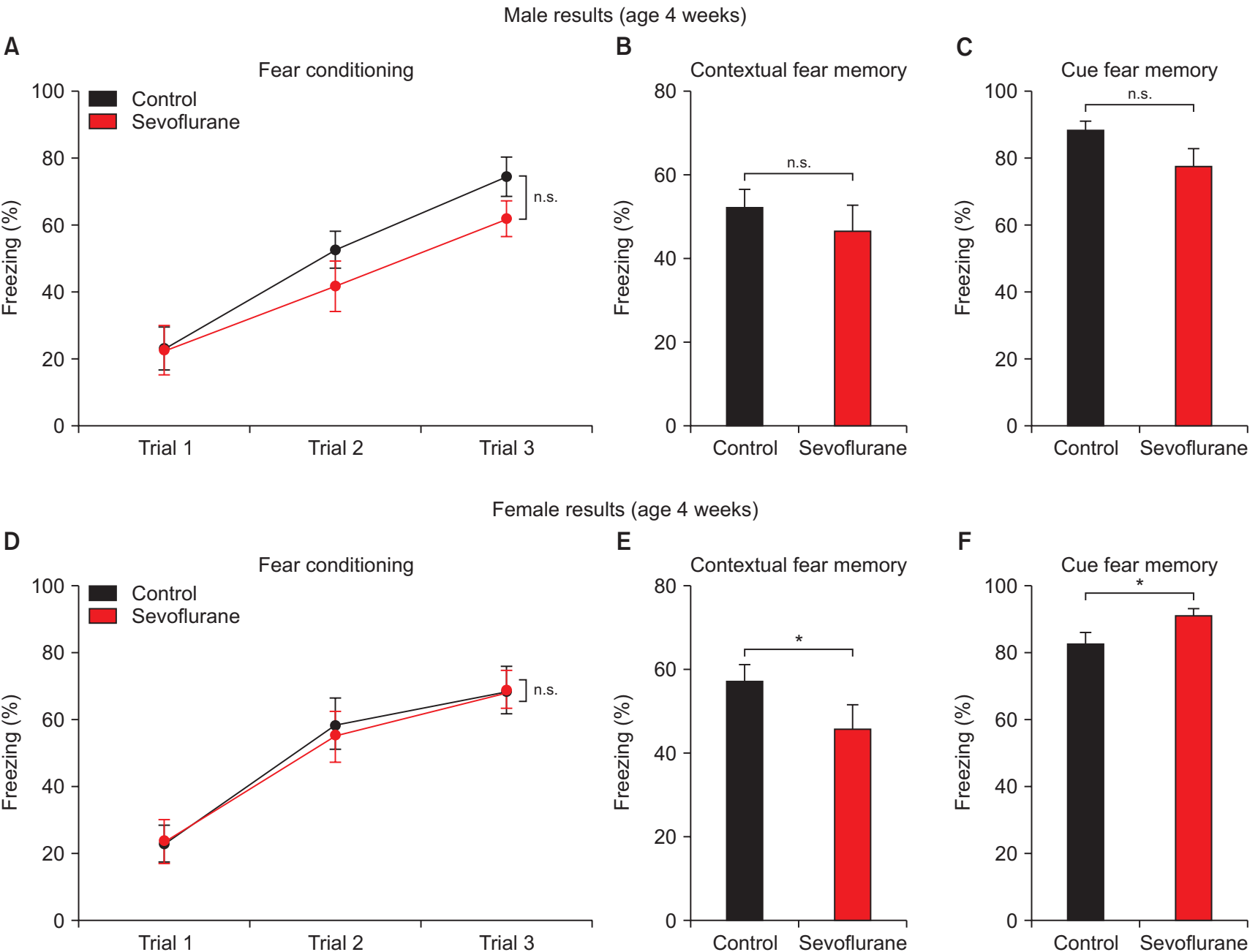

Fig. 2. Multiple sevoflurane exposures during pregnancy affect learning and memory sex-dependently in young offspring mice. (A-C) Fear chamber test results of young male mice show no differences after multiple sevoflurane exposures (linear regression for fear conditioning, $\mathrm{P}=0.340$; Student's $t$ test for contextual fear memory, $\mathrm{P}=0.451$; Student's $t$ test for cue fear memory, $\mathrm{P}=0.107$; ns, not significant). $\mathrm{N}=11$ for both groups. Values are presented as mean \pm SEM. (D-F) Fear chamber test results of young female mice show significant changes after multiple sevoflurane exposures. While there is no change during fear conditioning, sevoflurane exposed mice show a significantly decrease of contextual memory and increase of cue memory (linear regression for fear conditioning, $\mathrm{P}=0.978$; Kruskall-Wallis test for contextual fear memory, $\mathrm{P}=0.037$; Student's $t$ test for cue fear memory, $\mathrm{P}=0.038 ;{ }^{*} \mathrm{P}<0.05$; ns, not significant). $\mathrm{N}=10,13$ for control and sevoflurane groups, respectively. Values are presented as mean $\pm \mathrm{SEM}$.

[7,11-14]. Secondly, learning and memory impairments after early exposure have been shown to be reversible by raising the mice in a stimulating environment [10]. It is possible that anesthesia-induced cognitive impairments may self-resolve as the mice continue to grow and develop. However, multiple sevoflurane exposures during the fetal period did not induce changes in young offspring mice (Fig. 1).

Neurodevelopment is dependent on many factors. Although often disregarded, sex greatly influences diverse biological functions including neurodevelopment [20-22]. Interestingly, sexual dimorphic neurodevelopment has also been implied to be involved with neurodevelopmental disorders and various neurotoxic insults [21,23-26], including neurotoxicity from anesthesia
$[15,16,27,28]$. Thus, we next examined how sexually dimorphic neurodevelopment affects learning and memory after multiple sevoflurane exposures in pregnant mice (Fig. 2). Interestingly, our results suggest that early sevoflurane exposures during the fetal period impairs long-term contextual memory only in young female mice, which agrees with a previous study suggesting that female mice may be more vulnerable to anesthesiainduced neurotoxicity [27]. Thus, our study emphasizes the importance of age and sex when studying the long-term effects of sevoflurane exposures. However, our results also show that cue-fear memory is increased in young female mice who received early anesthesia, complicating the interpretation of our data. Since estrogen has been shown to affect cue-fear memory, 
A

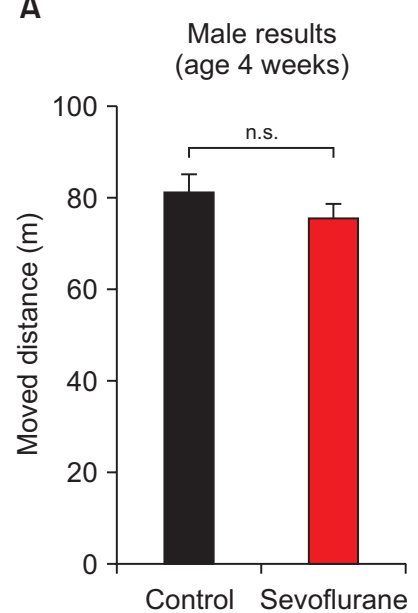

B

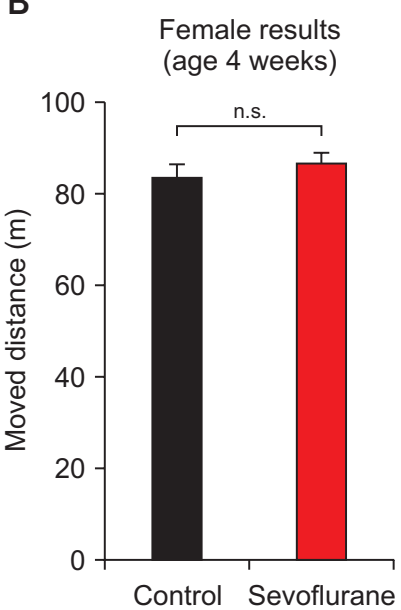

Fig. 3. Multiple sevoflurane exposures do not affect general activity in young mice. (A, B) Total moved distance of young male $(\mathrm{N}=11$ for both groups) and female mice ( $\mathrm{N}=10,13$ for control and sevoflurane groups, respectively) in the open field test is not affected by early anesthetic exposures (Student's $t$ test for male mice, $P=0.280$, Student's $\mathrm{t}$ test for female mice, $\mathrm{P}=0.370$; ns, not significant). Values are presented as mean \pm SEM.

our conflicting results may be due to the influence of the estrous cycle [29], which can occur by PND 30 [30]. Unfortunately, we are presently unable to present a clear explanation why such differences occurred.

There are several limitations in the present study. Although we are now able to suggest that age does not affect fear chamber tests in male mice after early anesthesia, we are still unable to suggest the same in female mice. It is still possible that older female mice may show impaired learning and memory. Also, we still do not know if our results can be replicated in other learning and memory behavioral assays such as the Morris water maze.

In conclusion, multiple sevoflurane exposures in the second trimester of pregnancy affects learning and memory only in young female mice. We suggest that age and sex must be considered when examining long-term effects of early exposures to anesthetics. As great interests are being focused on gender differences in many clinical fields, more detailed study designs focusing on the sexual difference in the form of inflammatory markers, cytokines, hormonal levels, and neurodevelopment may help understand the sex-dependent findings of the present study.

\section{Acknowledgments}

The present research was supported by the National Research Foundation of Korea (grant no. NRF-2015R1C1A1A01054659; Daejeon, Korea).

\section{References}

1. Lin EP, Lee JR, Lee CS, Deng M, Loepke AW. Do anesthetics harm the developing human brain? An integrative analysis of animal and human studies. Neurotoxicol Teratol 2017; 60: 117-28.

2. Koo BN. Anesthetic induced neurotoxicity in children. Korean J Anesthesiol 2017; 70: 237-8.

3. Davidson AJ, Disma N, de Graaff JC, Withington DE, Dorris L, Bell G, et al. Neurodevelopmental outcome at 2 years of age after general anaesthesia and awake-regional anaesthesia in infancy (GAS): an international multicentre, randomised controlled trial. Lancet 2016; 387: 239-50.

4. Sun LS, Li G, Miller TL, Salorio C, Byrne MW, Bellinger DC, et al. Association between a single general anesthesia exposure before age 36 months and neurocognitive outcomes in later childhood. JAMA 2016; 315: 2312-20.

5. Andropoulos DB, Greene MF. Anesthesia and developing brains - implications of the FDA warning. N Engl J Med 2017; 376: 905-7.

6. Silbereis JC, Pochareddy S, Zhu Y, Li M, Sestan N. The cellular and molecular landscapes of the developing human central nervous system. Neuron 2016; 89: 248-68.

7. Fang F, Song R, Ling X, Peng M, Xue Z, Cang J. Multiple sevoflurane anesthesia in pregnant mice inhibits neurogenesis of fetal hippocampus via repressing transcription factor Pax6. Life Sci 2017; 175: 16-22.

8. Workman AD, Charvet CJ, Clancy B, Darlington RB, Finlay BL. Modeling transformations of neurodevelopmental sequences across mammalian species. J Neurosci 2013; 33: 7368-83.

9. Palanisamy A, Baxter MG, Keel PK, Xie Z, Crosby G, Culley DJ. Rats exposed to isoflurane in utero during early gestation are behaviorally abnormal as adults. Anesthesiology 2011; 114: 521-8.

10. Zheng H, Dong Y, Xu Z, Crosby G, Culley DJ, Zhang Y, et al. Sevoflurane anesthesia in pregnant mice induces neurotoxicity in fetal and offspring mice. Anesthesiology 2013; 118: 516-26.

11. Suehara T, Morishita J, Ueki M, Ueno M, Maekawa N, Mizobuchi S. Effects of sevoflurane exposure during late pregnancy on brain development of offspring mice. Paediatr Anaesth 2016; 26: 52-9.

12. Lee S, Chung W, Park H, Park H, Yoon S, Park S, et al. Single and multiple sevoflurane exposures during pregnancy and offspring behavior in mice. Paediatr Anaesth 2017; 27: 742-51. 
13. Zhao T, Li Y, Wei W, Savage S, Zhou L, Ma D. Ketamine administered to pregnant rats in the second trimester causes long-lasting behavioral disorders in offspring. Neurobiol Dis 2014; 68: 145-55.

14. Kong FJ, Tang YW, Lou AF, Chen H, Xu LH, Zhang XM, et al. Effects of isoflurane exposure during pregnancy on postnatal memory and learning in offspring rats. Mol Biol Rep 2012; 39: 4849-55.

15. Rothstein S, Simkins T, Nuñez JL. Response to neonatal anesthesia: effect of sex on anatomical and behavioral outcome. Neuroscience 2008; 152: 959-69.

16. Lee BH, Chan JT, Kraeva E, Peterson K, Sall JW. Isoflurane exposure in newborn rats induces long-term cognitive dysfunction in males but not females. Neuropharmacology 2014; 83: 9-17.

17. Chung W, Park S, Hong J, Park S, Lee S, Heo J, et al. Sevoflurane exposure during the neonatal period induces long-term memory impairment but not autism-like behaviors. Paediatr Anaesth 2015; 25: 1033-45.

18. Chung W, Ryu MJ, Heo JY, Lee S, Yoon S, Park H, et al. Sevoflurane Exposure during the Critical Period Affects Synaptic Transmission and Mitochondrial Respiration but Not Long-term Behavior in Mice. Anesthesiology 2017; 126: 288-99.

19. Hu D, Flick RP, Zaccariello MJ, Colligan RC, Katusic SK, Schroeder DR, et al. Association between exposure of young children to procedures requiring general anesthesia and learning and behavioral outcomes in a population-based birth cohort. Anesthesiology 2017; 127: 227-40.

20. Dewing P, Shi T, Horvath S, Vilain E. Sexually dimorphic gene expression in mouse brain precedes gonadal differentiation. Brain Res Mol Brain Res 2003; 118: 82-90.

21. Mergler D. Neurotoxic exposures and effects: gender and sex matter! Hänninen Lecture 2011. Neurotoxicology 2012; $33: 644-51$.

22. Klein SL, Flanagan KL. Sex differences in immune responses. Nat Rev Immunol 2016; 16: 626-38.

23. Werling DM, Parikshak NN, Geschwind DH. Gene expression in human brain implicates sexually dimorphic pathways in autism spectrum disorders. Nat Commun 2016; 7: 10717.

24. Vahter M, Gochfeld M, Casati B, Thiruchelvam M, Falk-Filippson A, Kavlock R, et al. Implications of gender differences for human health risk assessment and toxicology. Environ Res 2007; 104: 70-84.

25. Mayoral SR, Omar G, Penn AA. Sex differences in a hypoxia model of preterm brain damage. Pediatr Res 2009; 66: 248-53.

26. Bronson SL, Chan JC, Bale TL. Sex-specific neurodevelopmental programming by placental insulin receptors on stress reactivity and sensorimotor gating. Biol Psychiatry 2017; 82: 127-38.

27. Boscolo A, Ori C, Bennett J, Wiltgen B, Jevtovic-Todorovic V. Mitochondrial protectant pramipexole prevents sex-specific long-term cognitive impairment from early anaesthesia exposure in rats. Br J Anaesth 2013; 110 Suppl 1: i47-52.

28. Gonzales EL, Yang SM, Choi CS, Mabunga DF, Kim HJ, Cheong JH, et al. Repeated neonatal propofol administration induces sexdependent long-term impairments on spatial and recognition memory in rats. Biomol Ther (Seoul) 2015; 23: 251-60.

29. Morgan MA, Pfaff DW. Effects of estrogen on activity and fear-related behaviors in mice. Horm Behav 2001; 40: 472-82.

30. Novaira HJ, Yates M, Diaczok D, Kim H, Wolfe A, Radovick S. The gonadotropin-releasing hormone cell-specific element is required for normal puberty and estrous cyclicity. J Neurosci 2011;31:3336-43. 